\title{
Dendrite growth velocity in levitated undercooled nickel melts
}

\author{
O. Funke ${ }^{\mathrm{a}}$, G. Phanikumar ${ }^{\mathrm{a}, \mathrm{c}}$, P.K. Galenko ${ }^{\mathrm{a}, *}$, L. Chernova $^{\mathrm{a}}$, S. Reutzel ${ }^{\mathrm{a}, \mathrm{b}}$, M. Kolbe $^{\mathrm{a}}$, \\ D.M. Herlach ${ }^{\mathrm{a}}$ \\ ${ }^{\mathrm{a}}$ German Aerospace Center (DLR), Institute of Space Simulation, 51170 Cologne, Germany \\ ${ }^{\mathrm{b}}$ Ruhr-University, Institute of Experimental Physics IV, 44780 Bochum, Germany \\ ${ }^{\mathrm{c}}$ Department of Metallurgical and Materials Engineering, Indian Institute of Technology Madras, Chennai, India
}

Received 21 February 2006; accepted 30 August 2006

Communicated by T.F. Kuech

Available online 18 October 2006

\begin{abstract}
Model predictions for the dendrite growth velocity at low undercoolings are deviating significantly from experimental data obtained in electromagnetic levitation with a capacitance proximity sensor (CPS) [K. Eckler, D.M. Herlach, Mater. Sci. Eng. A 178 (1994) 159]. In addition to that, previous data sets obtained by different techniques are not in good agreement with each other. For instance, growth velocity data for nickel melts obtained with a high-speed camera system [D.M. Matson, in: Solidification 1998, TMS, Warrendale PA, 1998, p. 233] show higher values at low undercoolings than data obtained with the CPS. Within this work new measurements of dendritic growth velocity in levitated undercooled nickel samples were performed as a function of undercooling $\Delta T$ to investigate this discrepancy. Solidification of the undercooled melt was detected at undercooling levels within the range of $30 \mathrm{~K}<\Delta T<300 \mathrm{~K}$. The new data reveal high accuracy and low scattering. These data are compared with two independent growth velocity data sets and discrepancies are discussed. For verification of the new CPS data dendrite growth velocity was also measured by using a high-speed camera where the morphology of the intersection of the solidification front with the sample surface was investigated. The new experimental data are analyzed within the model of dendrite growth obtained on the basis of Brener's theory [E. Brener, J. Crystal Growth 99 (1990) 165] and the model of dendrite growth with melt convection in a solidifying levitated drop, presently being developed. Special attention is paid to the effects of convection and small amounts of impurities on the growth dynamics at small undercoolings.
\end{abstract}

(C) 2006 Elsevier B.V. All rights reserved.

PACS: $05.70 . \mathrm{Fh} ; 68.70 .+\mathrm{w} ; 81.10 . \mathrm{Aj}$

Keywords: A1. Dendritic solidification; A1. Electromagnetic levitation; A1. Growth rate; A1. Undercooling

\section{Introduction}

Since several decades dendritic growth velocities $V$ have been measured during solidification of electromagnetically levitated metal melts. Different techniques have been applied, e.g. the usage of a fast responding photo-doublediode [1,2] or a high-speed camera system [3]. Following the sharp interface model of dendrite growth by Kurz et al. (LGK/LKT model) [4,5], the prediction of dendritic

\footnotetext{
*Corresponding author. Tel.: + 4922036014582 ; fax: + 4922036012255 .

E-mail address: peter.galenko@dlr.de (P.K. Galenko).
}

growth velocity $V$ for nickel as a function of undercooling $\Delta T$ is in good agreement with experimental data only in the region of medium undercoolings $(100 \mathrm{~K}<\Delta T<200 \mathrm{~K})$ [2]. However, for low undercoolings $(\Delta T<100 \mathrm{~K})$ the LGK/ LKT model is not able to describe the experimental data: the predicted growth velocity is too low and the discrepancy is actually increasing with decreasing $\Delta T[2,6]$.

The present investigation concentrates on the discrepancy occurring especially at low undercoolings. As already suggested [6], convection rolls occur in drops of pure $\mathrm{Ni}$ due to stirring of the melt during electromagnetic levitation. The fluid flow direction of convection rolls can be upwards in the vicinity of the surface and downwards in the middle of the 